\title{
Effects of Elasticity Number on the Heated Flow of Viscoelastic Fluid inside Planar Channel with a Symmetric Abrupt Expansion
}

\author{
Amin Shahbani Zahiri ${ }^{1}$, Hassan Hassanzadeh ${ }^{1}$, Mohammad Mohsen Shahmardan $^{2, *}$, Mahmood Norouzi $^{2}$ \\ ${ }^{1}$ Department of Mechanical Engineering, University of Birjand, Iran \\ ${ }^{2}$ Department of Mechanical Engineering, Shahrood University of Technology, Iran
}

Copyright $\mathrm{O} 2017$ by authors, all rights reserved. Authors agree that this article remains permanently open access under the terms of the Creative Commons Attribution License 4.0 International License

\begin{abstract}
In this work, we have numerically studied the heat transfer of viscoelastic fluid flow inside a planar channel with 1:3 abrupt expansion. For modeling rheological behavior of non-creeping flow related to the viscoelastic fluid which includes both the effects of shear-thinning and elasticity, the constitutive equation of exponential Phan Thien-Tanner (EPTT) is used. Also, thermal boundary condition of constant temperature at the walls and inlet of channel with internal heat generated by the viscous dissipation are considered. Because of high temperature differences in the current study, the fluid properties such as fluid viscosity, relaxation time, specific heat capacity and thermal conductivity have a function of temperature. For coupling the variables of velocity, pressure and temperature, the PISO algorithm is employed and finite volume method (FVM) imposed on the collocated grid to discretize the governing equations. The main propose of the present study is to examine the effects of elastic property on the size of recirculation regions, temperature distribution, local and mean Nusselt numbers.
\end{abstract}

Keywords Abrupt Expansion, Recirculation Region, Nusselt Number, Elasticity Number

\section{Introduction and Goals}

The flow of non-Newtonian fluids has a broad range of applications in petrochemical, chemical, pharmaceutical, Foodstuffs, paint and plastics industries. Also, in many of these industries, non-Newtonian fluid flow is non-isothermal and in some of them, the used chemical materials are melted (such as polymer melt flow in the plastics processing) and have a high temperature. In plastics processing, polymer melts demonstrate rheological behaviors typical of
non-Newtonian fluids especially viscoelastic fluids that this behavior is very dependent on the temperature and shear strain rate. Moreover, the transport of non-isothermal polymeric liquid products often occurs under laminar flow condition inside circular or square channels which the common parts of the channels are expansion or contraction and changes in cross-sectional area can be abrupt or non-abrupt. In recent decades, many researchers have studied isothermal and non-isothermal non-Newtonian fluid flow in straight pipe and channel. Also, several studies have been done for isothermal non-Newtonian fluid flow through a channel with abrupt and gradual expansion while the literature on non-isothermal state related to this class of problems are scarce.

Coelho et al. [1] carried out a theoretical study on the Graetz problem for viscoelastic fluid flow inside planar and axisymmetric channels with constitutive equation of Phan Thien-Tanner (PTT). Nóbrega et al. [2] have numerically and theoretically studied viscoelastic fluid flow and heat transfer by a rheological constitutive equation of linear Phan Thien-Tanner (LPTT). Alves et al. [3] investigated the fully developed laminar flow of generalized Newtonian fluid (such as Casson, Carreau-Yasuda, Herschel-Bulkley and Bingham models) and viscoelastic fluid (such as simplified Phan Thien-Tanner model) inside the pipe with a constant wall temperature. Letelier et al. [4] analytically solved the Graetz problem for viscoelastic fluid flow in a straight channel with arbitrary cross-section. They employed the modified Phan Thien-Tanner (MPTT) constitutive equation to investigate secondary flows, temperature distribution and Nusselt number.

Vaz \& Zdanski [5] used fully implicit finite difference scheme to examine creeping flow of polymer melt inside planar channel and asymmetric sudden expansion (backward-facing step) by the Cross constitutive equation. Zdanski \& Vaz Jr. [6, 7] investigated the non-Newtonian behavior of polymer melt creeping flow in asymmetric 
sudden expansion by constitutive equations of Cross and modified Arrhenius power-law. They examined the effects of inlet temperature and expansion ratio on the pressure loss coefficient and showed that the pressure loss is descended when the value of expansion ratio is decreased from 4 to 2 .

According to the past studies, it can be concluded that the majority of these studies has concentrated on the heat transfer of creeping flow related to the viscoelastic fluids inside the planar channel with asymmetric abrupt changes of cross-sectional area at low Weissenberg number and low temperature difference. Therefore, it is clear that examination of the non-creeping flow of heated viscoelastic fluid trough the planar channel with the symmetric abrupt expansion is important especially with considering the non-linear rheological models, high temperature difference and temperature-dependent properties in the simulation.

The schematic of planar channel with a symmetric 1:3 abrupt expansion has been indicated in Fig. 1. According to the Fig. 1, the lengths and heights of the upstream region (first part) and downstream region (second part) of the channel are respectively named as $\mathrm{L}_{1}, \mathrm{~h}$ and $\mathrm{L}_{2}, \mathrm{H}$. The purposes of the present study are to investigate the effects of elasticity number on the size of recirculation regions, temperature distribution and variation of the local and mean Nusselt numbers. Therefore, the heat transfer related to the non-creeping flow of viscoelastic fluid with an extremely elastic viscosity $(\beta=1 / 15)$ is simulated through the planar channel with a symmetric 1:3 abrupt expansion for different elasticity numbers $(0.01 \leq \mathrm{El} \leq 2)$ at $\mathrm{Re}=50, \mathrm{Br}=1$ and $\mathrm{Pr}=1$.

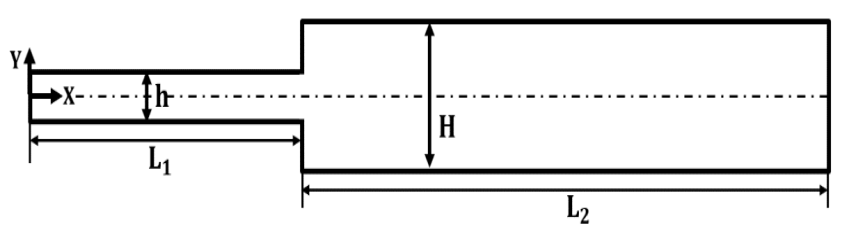

Figure 1. Schematic plan of the planar channel with a symmetric $1: 3$ abrupt expansion

\section{Numerical Method}

In this study, the viscoelastic fluid flow has been simulated inside a planar channel with a symmetric $1: 3$ abrupt expansion by OpenFOAM software. For discretizing the governing equations on the collocated grid, the finite volume method (FVM) has been used [8] and the PISO algorithm is employed for coupling pressure, velocity and temperature. Discretizing the convection term in the governing equations is carried out by the linear-upwind differencing scheme (LUDS) and the terms of gradient and Laplacian are discretized by the central difference [9]. The values of cell face are determined by second-order linear interpolation and volume integrals are calculated by second-order Gaussian integration. The algebraic equations system created by the discretization of governing equations is solved via the conjugated gradient (CG) method for pressure (which this method employed the AMG type as preconditioner) and the BiCGstab method for velocity components, stress tensor and temperature with a Cholesky preconditioner [10].

At the inlet of channel, boundary conditions are included the constant values for uniform velocity and temperature and the zero gradient for pressure and stress. Moreover, at the outlet of the channel, the relative pressure is zero and the gradients of the velocity, temperature and stress are considered to be zero. The no-slip conditions are applied for the velocity of fluid along the channel walls. Also, perpendicular to the channel wall, the gradient values of pressure and corrected stress tensor components are zero. Value of temperature is uniform and constant at the walls of the channel. In the following, the algorithm of numerical solution is expressed briefly [11]:

1. Discretizing the governing equations and set the defined boundary conditions.

2. Calculating the intermediate velocity components and the mass fluxes at the cell faces.

3. Solving the pressure equation for achieving the modified mass fluxes for the cell faces.

4. Recalculation the velocity components by the new pressure field.

5. Calculating pressure equation and modifying velocity components by the new pressure field for second time.

6. Solve the constitutive equation of polymeric stress and update the temperature-dependent properties.

7. Solve the energy equation and Update the defined boundary conditions.

8. Repeats the solution again from step 2 until the convergence criterion is satisfied.

\subsection{Equations}

For the numerical simulation of laminar flow related to the incompressible viscoelastic fluid inside the planar channel with a symmetric abrupt expansion, the equations of continuity, momentum and thermal energy to be solved as follows:

$$
\begin{gathered}
\frac{\partial u_{i}}{\partial x_{i}}=0 \\
\frac{\partial\left(\rho u_{i}\right)}{\partial t}+\frac{\partial\left(\rho u_{j} u_{i}\right)}{\partial x_{j}}=-\frac{\partial p}{\partial x_{i}}+\frac{\partial \tau_{i j}^{S}}{\partial x_{j}}+\frac{\partial \tau_{i j}^{P}}{\partial x_{j}} \\
\frac{\partial\left(\rho C_{p} T\right)}{\partial t}+\frac{\partial\left(\rho c_{p} u_{j} T\right)}{\partial x_{j}}=\frac{\partial}{\partial x_{j}}\left(k_{f} \frac{\partial T}{\partial x_{j}}\right)+\tau_{i j}^{S} \frac{\partial u_{i}}{\partial x_{j}}+\tau_{i j}^{P} \frac{\partial u_{i}}{\partial x_{j}},
\end{gathered}
$$

where $u_{i}, \rho, p$ represent the velocity vector, density and pressure respectively. $\mathrm{T}$ is the temperature, $k_{f}$ is the thermal conductivity and $\mathrm{C}_{\mathrm{p}}$ shows the specific heat capacity. Also, $\tau_{i j}^{P}$ and $\tau_{\mathrm{ij}}^{\mathrm{S}}$ are the polymeric stress and the Newtonian solvent stress, which Newtonian stress is expressed by the Newton-law as follows: 


$$
\tau_{i j}^{S}=\eta_{s}\left(\frac{\partial u_{i}}{\partial x_{j}}+\frac{\partial u_{j}}{\partial x_{i}}\right),
$$

where the solvent viscosity is defined by $\eta_{s}$. For modeling the behavior of polymeric fluid in the present research, the exponential form of Phan Thien-Tanner (PTT) constitutive equation is used that it has suitable accuracy for simulation of the heated flow related to the materials such as concentrated solutions and polymeric melts [12]. The constitutive equation of Phan Thien-Tanner is indicated as [13]:

$$
\begin{aligned}
f\left(\operatorname{tr} \tau_{i j}^{P}\right) \tau_{i j}^{P} & +\lambda\left(u_{k} \frac{\partial \tau_{i j}^{P}}{\partial x_{k}}-\tau_{i k}^{P} \frac{\partial u_{j}}{\partial x_{k}}-\frac{\partial u_{i}}{\partial x_{k}} \tau_{k j}^{P}\right)+\frac{\xi}{2}\left[\tau_{i j}^{P}\left(\frac{\partial u_{i}}{\partial x_{j}}+\frac{\partial u_{j}}{\partial x_{i}}\right)+\left(\frac{\partial u_{i}}{\partial x_{j}}+\frac{\partial u_{j}}{\partial x_{i}}\right) \tau_{i j}^{P}\right] \\
& =2 \eta_{p}\left(\frac{\partial u_{i}}{\partial x_{j}}+\frac{\partial u_{j}}{\partial x_{i}}\right),
\end{aligned}
$$

Where $\eta_{\mathrm{p}}$ is the polymeric additive viscosity and $\xi$ is adjustable free parameter and the exponential form of stress coefficient function $f\left(\operatorname{tr} \tau_{i j}^{P}\right)$ is defined as follows [14]:

$$
f\left(\operatorname{tr} \tau_{i j}^{P}\right)=\exp \left(\frac{\varepsilon \lambda}{\eta_{p}} \operatorname{tr} \tau_{i j}^{P}\right),
$$

Where the parameters of $\varepsilon$ and $\lambda$ are extensibility property and relaxation time, respectively. In the present study, the values of the adjustable parameters and extensibility are considered to be $\xi=0.04$ and $\varepsilon=0.02$ (according to the study of Cruz \& Pinho [15]). In this study, the used significant dimensionless groups are defined as follows:

$$
\mathrm{El}=\frac{\lambda \eta_{0}}{\rho \mathrm{D}_{\mathrm{h}}^{2}}, \quad \mathrm{Nu}=\frac{\mathrm{h}_{\mathrm{f}} \mathrm{D}_{\mathrm{h}}}{\mathrm{k}_{\mathrm{f}}}, \quad \mathrm{T}^{*}=\frac{\mathrm{T}_{\mathrm{w}}-\mathrm{T}}{\mathrm{T}_{\mathrm{w}}-\mathrm{T}_{\mathrm{i}}}, \quad \mathrm{U}^{*}=\frac{\mathrm{U}}{\mathrm{U}_{\mathrm{i}}}, \quad \mathrm{X}^{*}=\frac{\mathrm{X}}{\mathrm{h}}, \quad \mathrm{Y}^{*}=\frac{\mathrm{Y}}{\mathrm{h}},
$$

where $\mathrm{El}$ and $\mathrm{Nu}$ are elasticity and Nusselt numbers respectively. Also, $\mathrm{T}^{*}, \mathrm{U}^{*}, \mathrm{X}^{*}$ and $\mathrm{Y}^{*}$ represent a dimensionless form related to the parameters of temperature $(\mathrm{T})$, velocity $(\mathrm{U})$, horizontal and vertical Cartesian coordinates $(\mathrm{X}, \mathrm{Y})$ respectively. Parameters of $h_{f}$ and $D_{h}$ represent heat transfer coefficient, and hydraulic diameter of channel $\left(D_{h}=2 h\right)$ respectively. Also, $U_{i}, T_{i}$ and $T_{w}$ indicate uniform velocity at channel inlet, constant temperatures at the inlet and walls of the channel respectively. Furthermore, total viscosity of viscoelastic fluid $\left(\eta_{0}\right)$ is achieved by the summation of solvent and polymeric additives viscosities at zero shear rate $\left(\eta_{0}=\eta_{\mathrm{S}}=\eta_{\mathrm{P}}\right)$. The elasticity parameter serves as a stabilizing factor for viscoelastic fluid flow with the ratio of solvent viscosity ( $\beta$ ) equal to 0.5 or higher, while for higher polymeric concentration $(\beta<<1)$, the elasticity parameter operates as an unstable factor in the event of bifurcation phenomena [16]. Therefore, the ratio of the solvent viscosity to the total viscosity is chosen to be very low $\left(\beta=\eta_{\mathrm{S}} /\left(\eta_{\mathrm{S}}+\eta_{\mathrm{P}}\right) \approx 1 / 15\right)$ to examine the behavior of viscoelastic fluid at very high polymeric viscosity.

In this study, the fluid properties such as fluid viscosity, relaxation time, specific heat capacity and thermal conductivity are varied with temperature and the dependence of these parameters on the temperature are represented as follows [17]:

$$
\eta_{0}(\mathrm{~T})=\eta_{0, \mathrm{i}} \mathrm{a}(\mathrm{T}), \lambda(\mathrm{T})=\lambda_{\mathrm{i}} \mathrm{a}(\mathrm{T}), \mathrm{k}_{\mathrm{f}}(\mathrm{T})=\mathrm{k}_{\mathrm{f}, \mathrm{i}}\left(\mathrm{k}_{\mathrm{f}, 0}^{*}+\mathrm{k}_{\mathrm{f}, \mathrm{s}}^{*} \mathrm{~T}\right), \mathrm{C}_{\mathrm{p}}(\mathrm{T})=\mathrm{C}_{\mathrm{p}, \mathrm{i}}\left(\mathrm{C}_{\mathrm{p}, 0}^{*}+\mathrm{C}_{\mathrm{p}, \mathrm{s}}^{*} \mathrm{~T}\right),
$$


Where $\eta_{0, \mathrm{i}}, \lambda_{\mathrm{i}}, \mathrm{k}_{\mathrm{f}, \mathrm{i}}$ and $\mathrm{C}_{\mathrm{p}, \mathrm{i}}$ show the total fluid viscosity, relaxation time, thermal conductivity and specific heat capacity at the inlet temperature of channel. Also, a(T) is a shift factor and defined by the following Arrhenius function [17]:

$$
\mathrm{a}(\mathrm{T})=\exp \left[\alpha\left(\frac{1}{\mathrm{~T}+273.15}-\frac{1}{\mathrm{~T}_{0}+273.15}\right)\right]
$$

where $\mathrm{T}_{0}$ is reference temperature and constant values related to the parameters utilized in the equations (7-9) have been indicated in Table 1.

\section{Results and Discussion}

The main objective of this study is to evaluate the effects of elastic property on the heated viscoelastic fluid flow inside planar channel with a symmetric 1:3 abrupt expansion. In order to better examine the effect of the rheological behavior related to the viscoelastic fluid on the heat transfer rate, the properties such as fluid viscosity, relaxation time, specific heat capacity and thermal conductivity are taken as temperature-dependent functions and viscous dissipation is exerted in the energy equation. Thus, streamlines, length of vortices, temperature distribution, local and mean Nusselt numbers are illustrated for different elasticity numbers $(0.01 \leq \mathrm{El} \leq 2)$ in a large temperature difference $\left(\Delta \mathrm{T}=\mathrm{T}_{\mathrm{w}}-\mathrm{T}_{\mathrm{i}}=100^{\circ} \mathrm{C}\right)$.

\subsection{Mesh Independency}

In this section, independence of numerical solution from computational grid is investigated by velocity distribution on centerline of channel with symmetric sudden expansion for inertial and non-isothermal flow of viscoelastic fluid. For this purpose, in Fig. 2, the velocity profiles are plotted along the centerline of channel for four different grids at $\mathrm{Br}=1$, $\mathrm{We}=10, \mathrm{Re}=60$. Also, the names of four different grids are M1, M2, M3 and M4 (coarse mesh to very fine mesh respectively).

Table 1. Values of constant parameters used in the equations (7-9) [17]

\begin{tabular}{|c|c|c|c|c|c|}
\hline $\mathrm{k}_{\mathrm{f}, 0}^{*}$ & $\mathrm{k}_{\mathrm{f}, \mathrm{s}}^{*}$ & $\mathrm{k}_{\mathrm{f}, \mathrm{i}}$ & $\alpha$ & $\eta_{0, \mathrm{i}}$ & $\mathrm{T}_{\mathrm{w}}$ \\
\hline 0.7753 & $0.00118\left(1 /{ }^{\circ} \mathrm{C}\right)$ & $0.0275(\mathrm{~W} / \mathrm{mK})$ & $1720(\mathrm{~K})$ & $4.07(\mathrm{~Pa} . \mathrm{s})$ & $290\left({ }^{\circ} \mathrm{C}\right)$ \\
\hline $\mathrm{C}_{\mathrm{p}, 0}^{*}$ & $\mathrm{C}_{\mathrm{p}, \mathrm{s}}^{*}$ & $\mathrm{C}_{\mathrm{p}, \mathrm{i}}$ & $\mathrm{T}_{0}$ & $\rho$ & $\mathrm{T}_{0}$ \\
\hline 1.2122 & $-0.00112\left(1 /{ }^{\circ} \mathrm{C}\right)$ & $0.007(\mathrm{~J} / \mathrm{kg} . \mathrm{K})$ & $190\left({ }^{\circ} \mathrm{C}\right)$ & $1226(\mathrm{~kg} / \mathrm{m} 3)$ & $190\left({ }^{\circ} \mathrm{C}\right)$ \\
\hline
\end{tabular}

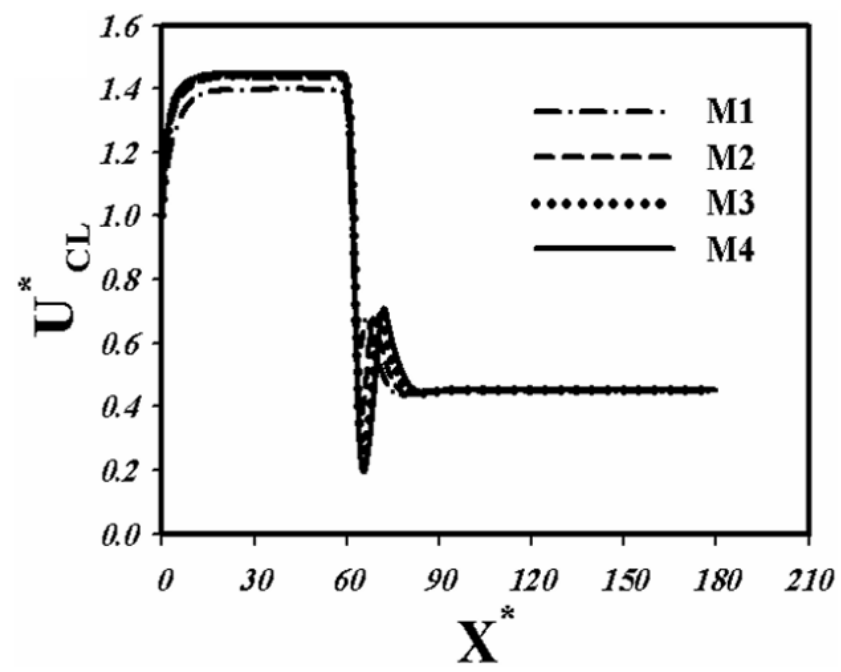

Figure 2. The velocity distribution of non-isothermal flow of viscoelastic fluid inside sudden expansion channel for four various grids along the centerline

Table 2. Characteristics of mesh cells for four different grids of sudden expansion channel.

\begin{tabular}{|c|c|c|c|c|c|}
\hline Grid & NMCY $_{1}$ & NMCY $_{2}$ & NMCX $_{1}$ & NMCX $_{2}$ & NMCT \\
\hline M1 & 6 & 18 & 140 & 400 & 8040 \\
\hline M2 & 10 & 30 & 224 & 640 & 21440 \\
\hline M3 & 16 & 48 & 358 & 1024 & 54880 \\
\hline M4 & 26 & 78 & 573 & 1638 & 142662 \\
\hline
\end{tabular}


The mesh cells characteristics of four different grids are given in detail in Table 2. According to Table 2, number of mesh cells in y-direction is shown by parameters of $\mathrm{NMCY}_{1}$ and $\mathrm{NMCY}_{2}$ for the first and second parts of planar channel respectively. Also, the number of mesh cells in X-direction is shown by parameters of $\mathrm{NMCX}_{1}$ and $\mathrm{NMCX}_{2}$ for first and second parts of planar channel respectively. The NMCT is the total number of mesh cells in the channel. As shown in Fig. 2, the grid M2 has a little difference for velocity profile in comparison with the grids M3 and M4, while velocity profiles related to the grids of M3 and M4 are almost identical. Therefore the grid M3 is a suitable choice for numerical investigation of current study.

\subsection{Verification}

In this section, the results related to the temperature distribution and velocity profile have been compared with previous researches to verify the numerical simulation. For this reason, viscoelastic fluid flow and heat transport has been simulated inside a straight planar channel with constitutive equation of simplified Phan Thien-Tanner (SPTT) to compare with past analytical results. First, velocity profile of viscoelastic fluid flow related to the numerical results of the current study (solid circle symbol) is compared with the analytical results of Oliveira \& Pinho [18] (solid line) at $\varepsilon=0.01, \mathrm{Re}=10$ and $\mathrm{We}=3$ that has been shown in Fig. 3. Also, comparison of temperature distribution in the current study (solid circle symbol) with analytical research of Coelho et al. [1] (solid line) has been indicated in Fig. 4 for $\operatorname{Re}=10, \operatorname{Pr}=100, \mathrm{Br}=-1$ and $\varepsilon \mathrm{We}^{2}=0.1$. It can be observed that comparison of the current study with previous researches corroborates a very good agreement.

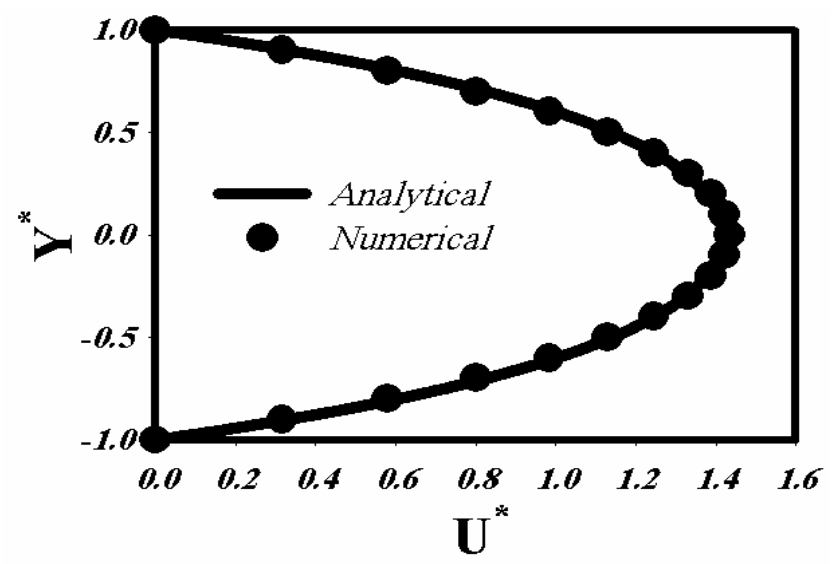

Figure 3. Comparison of the velocity profile in the current research with the analytical research of Oliveira \& Pinho [18] for flow of viscoelastic fluid at $\varepsilon=0.01, \mathrm{Re}=10$ and $\mathrm{We}=3$

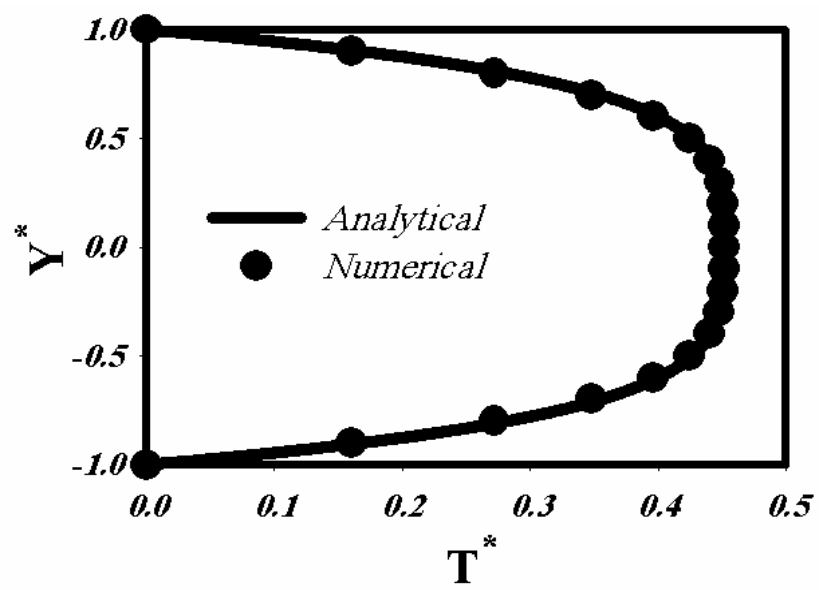

Figure 4. Comparison of the temperature distribution in the current research with the analytical research of Coelho et al. [1] for heat transfer of viscoelastic fluid flow at $\mathrm{Re}=10, \mathrm{Pr}=100, \mathrm{Br}=-1$ and $\varepsilon \mathrm{We}^{2}=0.1$

\subsection{Depiction and Description}

In Fig. 5, streamlines related to the heated flow of viscoelastic fluid are drawn for different elasticity numbers to represent the effects of elasticity property on the size of recirculation regions. Also for more details, bifurcation diagram related to the length of vortices (LV) has been indicated in Fig. 6. As it is shown in Fig. 6, the (V1) and (V3) branches demonstrated length of the first and second vortices in the vicinity of the lower and upper channel walls respectively. Also, the branches of (V2) and (V4) illustrated the start and end points of the third vortex adjacent to the lower channel wall. Thus, the distance between the branches of (V2) and (V4) represents the length of third vortex. As shown in the figs. 5 and 6 , as long as the elasticity number is sufficiently low $(\mathrm{El}=0.01)$, vortices are symmetric and by further growth of elasticity property, asymmetric vortices are created.

It is pertinent to mention that the symmetric and asymmetric steady states are based on the upstream axial velocity perturbation, the downstream convection of vorticity perturbation and the effects of viscous dissipation. When elasticity number (El) is incremented, the effects of upstream convection perturbation become dominant and, therefore, the asymmetric flow is created. On the other hand, by entering the viscoelastic fluid flow into the expanded part of the channel (second part), the fluid element is stretched perpendicular to the flow direction (y-direction) and compressed in the flow direction (x-direction), therefore, formation of fluid element is changed along the streamline and deformation history of fluid element is saved in the fluid memory. 
0.01

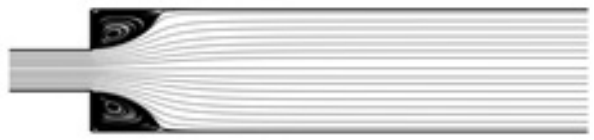

0.25

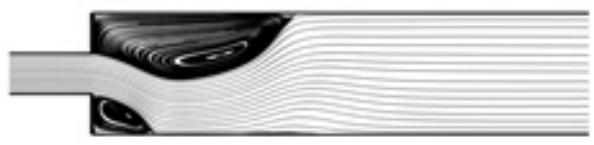

0.75

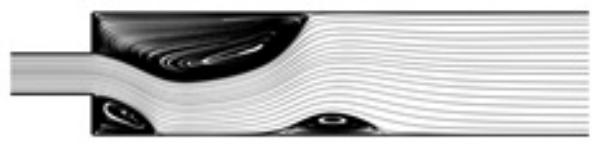

1.50

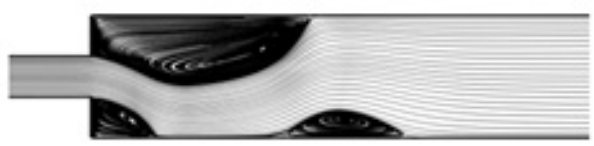

El

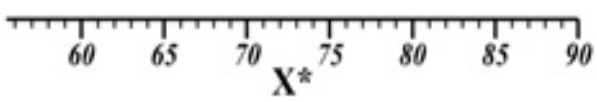

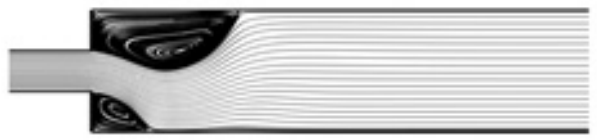

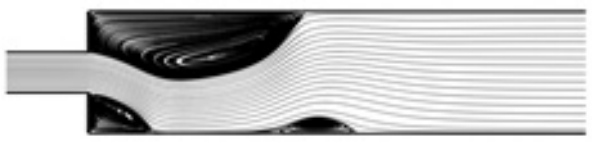

1.00

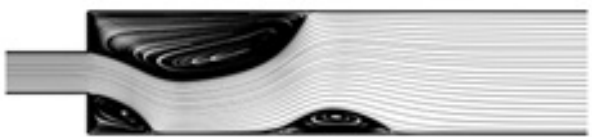

2.00

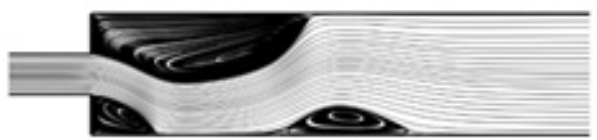

$\mathrm{El}$

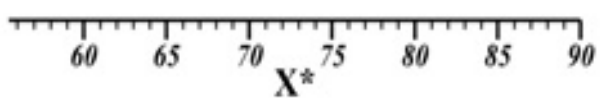

Figure 5. Streamlines related to the heated flow of viscoelastic fluid inside symmetric abrupt expansion channel for various elasticity numbers at Re=50, $\mathrm{Br}=1$ and $\mathrm{Pr}=1$

By increasing the elasticity number, the effects of perturbation and molecular deformation would be more that it causes the growth of vortices length. In asymmetric flow, the streamline is initially diverted to the channel lower wall, and then moved to the channel upper wall by formation of the third vortex. Therefore, the first vortex (in the vicinity of lower wall) and second vortex (in the vicinity of upper wall) are constant for the elasticity number greater than 0.5 and 0.75 respectively that it is due to the dominance of viscosity dissipation and vortices perturbation on the upstream convection perturbation. Also, the changes related to the length of third vortex shows that its variation is insignificant in the elasticity numbers greater than 2. Fig. 7 displays the temperature difference between the viscoelastic fluid flow and the channel walls in the vicinity of the upper and lower walls and along the centerline of channel. Due to the effects of fluid memory and viscous dissipation, the temperature of viscoelastic fluid flow throughout the expanded part of channel is higher than the constant temperature of the channel walls. By creating the recirculation regions in adjacent to the lower and upper walls, diagram curves fall down and after completion of the recirculation regions began to climb.

These minimum points of the diagram represent the end points related to the first and second vortices that at these points, the temperature of viscoelastic fluid flow is maximum in adjacent to the channel walls. Also, by incrementing the elastic property, the memory effect of fluid overcomes to the viscous dissipation influences, thus, amount of temperature difference between viscoelastic fluid flow and channel walls is decreased. In Fig. 8, the distributions of local Nusselt number related to the upper and lower walls of channel have been drawn at various elasticity numbers. In viscoelastic fluids with very high polymeric viscosity, by increasing the elastic property, the asymmetric flow is created inside planar channel with a symmetric abrupt expansion. Due to the formation of recirculation regions in the vicinity of the channel walls, the distribution of temperature and Nusselt number are different in adjacent to the lower and upper walls of the channel.

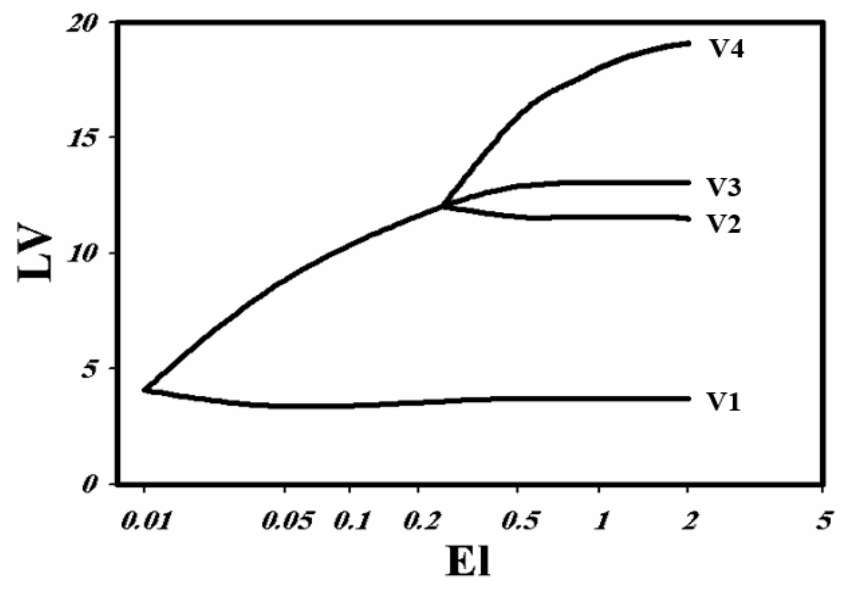

Figure 6. Length of vortices versus the different elasticity numbers for heated flow of viscoelastic fluid inside planar channel with a symmetric abrupt expansion at $\mathrm{Re}=50, \mathrm{Br}=1$ and $\mathrm{Pr}=1$. 

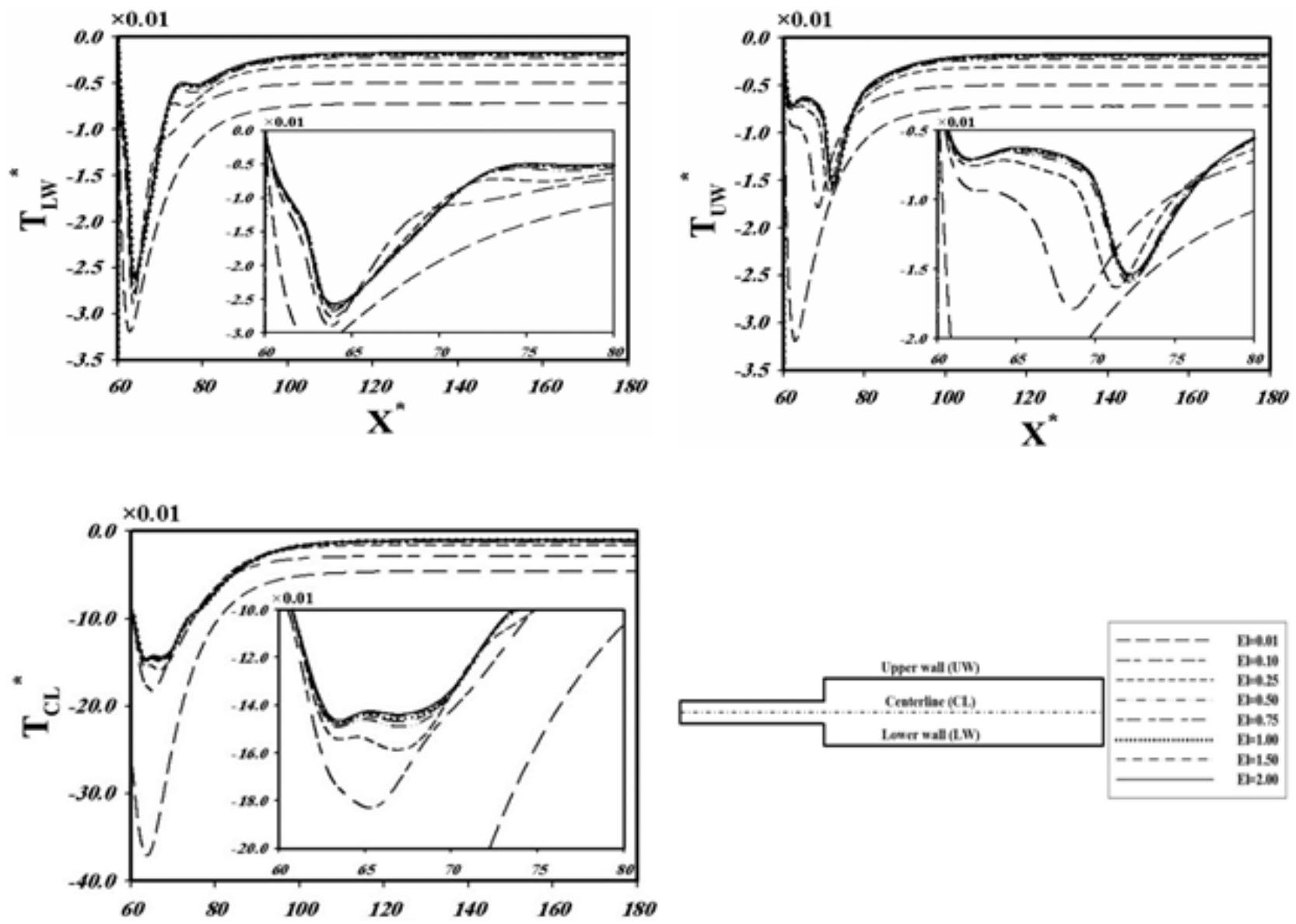

Figure 7. Temperature distribution along the centerline and in adjacent to the upper and lower walls of channel for different elasticity numbers at $\mathrm{Re}=50$, $\mathrm{Pr}=1$ and $\mathrm{Br}=1$
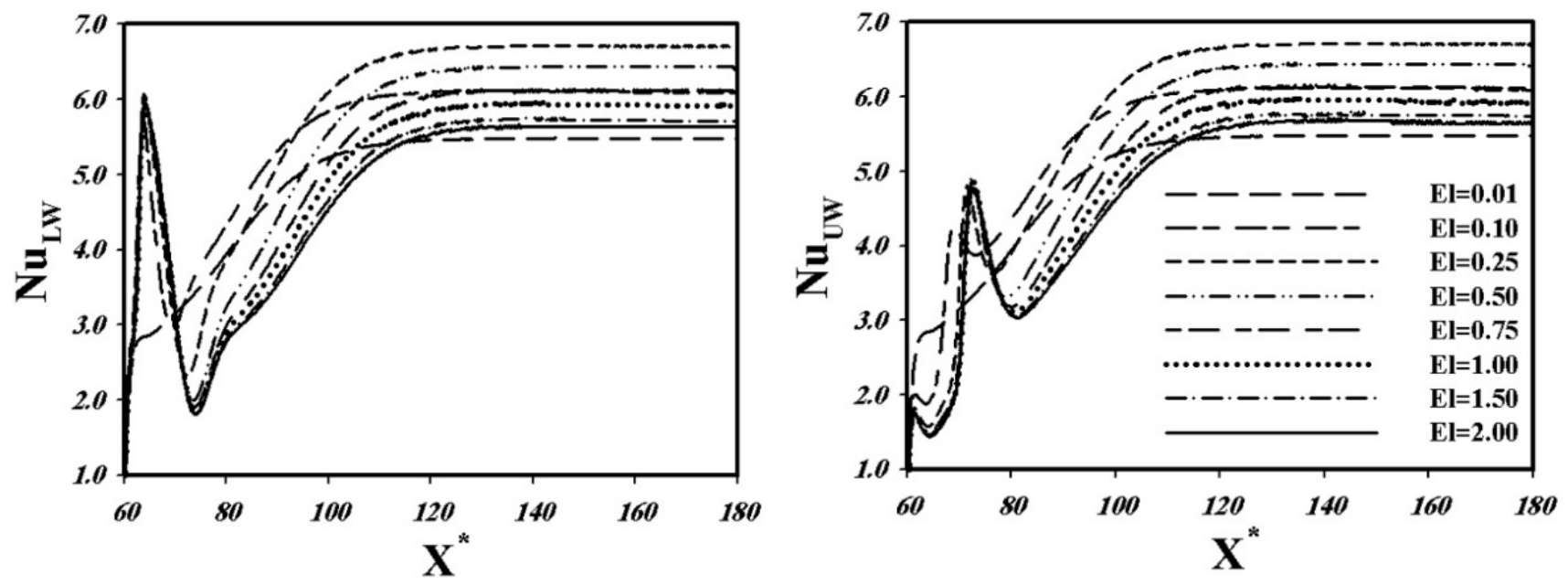

Figure 8. Distribution of local Nu number along the lower and upper walls of channel for different elasticity numbers at $\operatorname{Re}=50, \operatorname{Pr}=1$ and $\mathrm{Br}=1$

By forming the first vortex in the vicinity of the channel lower wall, the temperature difference between the channel wall and the viscoelastic fluid at its neighbor increases and thereby the local Nusselt number is incremented. Upon completion of first vortex, this temperature difference is reduced and consequently the Nusselt number of lower wall is descended and this reduction trending will continue till the end of the second vortex (in the vicinity of the upper wall). Then, it grows again, as long as, the heated flow achieve the thermal fully developed state and in this case, the local Nusselt number is fixed. The same event occurs for the local Nusselt number related to the channel upper wall. First, the local Nusselt number of the upper wall rises and with the end of the second vortex, it falls down to end of the third vortex 
(adjacent to the lower wall). Afterwards the local Nusselt number of the upper wall climbs up to reach the fixed value in thermal fully developed state.

Creating the asymmetry vortices and also, formation of third vortex has a large impact on the local and mean Nusselt numbers in the expanded part of channel. Prior to the formation of third vortex, growth of elastic property leading to the increase of local Nusselt numbers for the thermal fully developed flow (according to the Fig. 8) and mean Nusselt numbers in the expanded part of the channel (according to Fig. 9). With further growth of elasticity number and formation of third vortex in the vicinity of the lower wall, the local Nusselt number of thermal fully developed flow and mean Nusselt number related to the expanded part of the channel is decreased. Due to the formation of two vortices and creation of higher fluid temperature in adjacent to the channel lower wall in comparison with the temperature of fluid at the channel upper wall neighbor, the mean Nusselt number of lower wall is greater than the mean Nusselt number related to the upper wall for heated flow in throughout the expanded part of channel.

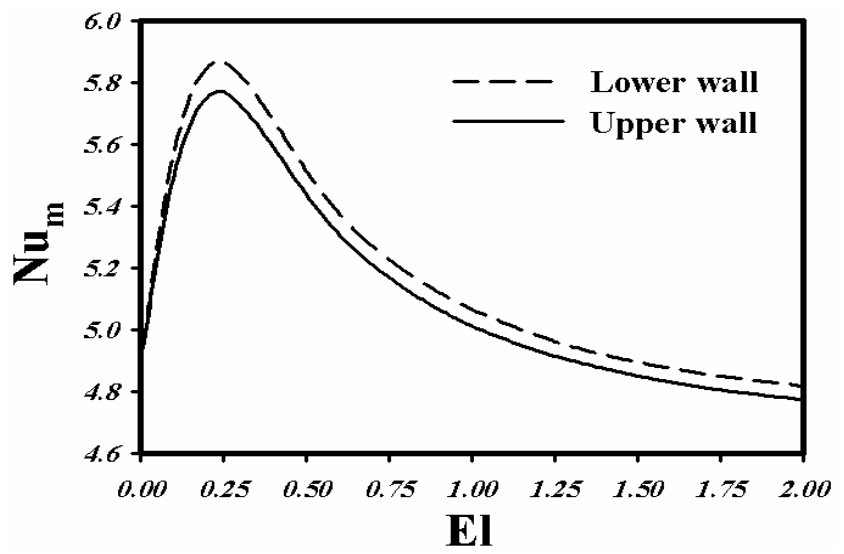

Figure 9. Distribution of mean $\mathrm{Nu}$ number throughout the expanded part of channel for various elasticity numbers at $\mathrm{Re}=50, \mathrm{Pr}=1$ and $\mathrm{Br}=1$

\section{Conclusions}

1. By considering the viscous dissipation for the heated flow of viscoelastic fluids with high polymeric viscosity, the temperature of fluid is higher than the constant wall temperature in throughout the expanded part of the channel.

2. In adjacent to the lower and upper walls of channel, maximum values of temperature are achieved at the end points related to the first and second vortices.

3. For thermal fully developed flow, maximum value of local Nusselt number is obtained before the formation of third vortex.

4. For heated flow of viscoelastic fluid inside the expanded part of channel, maximum value of mean Nusselt number is achieved before forming the third vortex.

\section{REFERENCES}

[1] P. M. Coelho, F. T. Pinho, and P. J. Oliveira, "Fully developed forced convection of the Phan-Thien-Tanner fluid in ducts with a constant wall temperature," International Journal of Heat and Mass Transfer, vol. 45, pp. 1413-1423, 2002.

[2] J. Nóbrega, F. T. d. Pinho, P. Oliveira, and O. Carneiro, "Accounting for temperature-dependent properties in viscoelastic duct flows," International Journal of Heat and Mass Transfer, vol. 47, pp. 1141-1158, 2004.

[3] M. A. Alves, A. Baptista, and P. M. Coelho, "Simplified method for estimating heat transfer coefficients: constant wall temperature case," Heat and Mass Transfer, vol. 51, pp. 1041-1047, 2015.

[4] M. F. Letelier, C. B. Hinojosa, and D. A. Siginer, "Analytical solution of the Graetz problem for non-linear viscoelastic fluids in tubes of arbitrary cross-section," International Journal of Thermal Sciences, vol. 111, pp. 369-378, 2017.

[5] M. Vaz Jr and P. S. B. Zdanski, "A fully implicit finite difference scheme for velocity and temperature coupled solutions of polymer melt flow," Communications in Numerical Methods in Engineering, vol. 23, pp. 285-294, 2007.

[6] P. S. B. Zdanski and M. Vaz Jr., "Three-dimensional polymer melt flow in sudden expansions: Non-isothermal flow topology," International Journal of Heat and Mass Transfer, vol. 52, pp. 3585-3594, 2009.

[7] P. S. B. Zdanski and M. Vaz Jr., "Non-isothermal polymer melt flow in sudden expansions," Journal of Non-Newtonian Fluid Mechanics, vol. 161, pp. 42-47, 2009.

[8] P. Oliveira, F. T. d. Pinho, and G. Pinto, "Numerical simulation of non-linear elastic flows with a general collocated finite-volume method," Journal of Non-Newtonian Fluid Mechanics, vol. 79, pp. 1-43, 1998.

[9] J. Favero, A. Secchi, N. Cardozo, and H. Jasak, "Viscoelastic flow analysis using the software OpenFOAM and differential constitutive equations," Journal of Non-Newtonian Fluid Mechanics, vol. 165, pp. 1625-1636, 2010.

[10] J. Lee, S. Yoon, Y. Kwon, and S. Kim, "Practical comparison of differential viscoelastic constitutive equations in finite element analysis of planar 4: 1 contraction flow," Rheologica Acta, vol. 44, pp. 188-197, 2004.

[11] H. K. Versteeg and W. Malalasekera, An introduction to computational fluid dynamics: the finite volume method, Second ed. Harlow, United Kingdom: Pearson Education Limited, 2007.

[12] L. Quinzani, R. Armstrong, and R. Brown, "Use of coupled birefringence and LDV studies of flow through a planar contraction to test constitutive equations for concentrated polymer solutions," Journal of Rheology, vol. 39, pp. 1201-1228, 1995.

[13] N. Phan-Thien and R. I. Tanner, "A new constitutive equation derived from network theory," Journal of Non-Newtonian Fluid Mechanics, vol. 2, pp. 353-365, 1977. 
[14] N. Phan-Thien, "A nonlinear network viscoelastic model," Journal of Rheology, vol. 22, pp. 259-283, 1978.

[15] D. Cruz and F. Pinho, "Fully-developed pipe and planar flows of multimode viscoelastic fluids," Journal of Non-Newtonian Fluid Mechanics, vol. 141, pp. 85-98, 2007.

[16] M. Norouzi, M. M. Shahmardan, and A. Shahbani Zahiri, "Bifurcation phenomenon of inertial viscoelastic flow through gradual expansions," Rheologica Acta, vol. 54, pp. 423-435,
2015.

[17] J. E. Mark, Physical properties of polymers handbook. New York: American Institute of Physics, 1996.

[18] P. J. Oliveira and F. T. Pinho, "Analytical solution for fully developed channel and pipe flow of Phan-Thien-Tanner fluids," Journal of Fluid Mechanics, vol. 387, pp. 271-280, 1999. 